\title{
Kinetic theory of simple reacting spheres: an application to coloring processes
}

\author{
Filipe Carvalho, Jacek Polewczak and Ana Jacinta Soares
}

\begin{abstract}
We consider a simplified version of the kinetic model of simple reacting spheres (SRS) for a quaternary reactive mixture of hard-spheres in the dilute-gas limit. The model mimics a coloring process occurring with probability $\alpha_{R}$, described by the reversible chemical law $A_{1}+A_{2} \rightleftharpoons A_{3}+A_{4}$. We provide the linearized collisional operators of our model and investigate some of their mathematical properties. In particular we obtain an explicit and symmetric representation of the elastic and reactive kernels and use this to prove the compactness of the linearized collisional operator in $\left(L^{2}\left(\mathbb{R}^{3}\right)\right)^{4}$.
\end{abstract}

\section{Introduction}

In these proceedings we continue the work developed by the authors in the context of the simple reacting spheres (SRS) model in the kinetic theory of chemically reacting gases (see [1] and [2]). The SRS kinetic theory has been initially proposed by Marron [3] and then developed by Xystris, Dahler and Qin in a series of papers devoted to the kinetic modeling, non-equilibrium processes, transport properties, and extension to dense-gas limit (see [4], [5] and [6]). Some advances related to the mathematical analysis of the SRS model have been done by Polewczak in paper [7], proving the existence of global-in-time, spatially inhomogeneous, and $L^{1}$ -

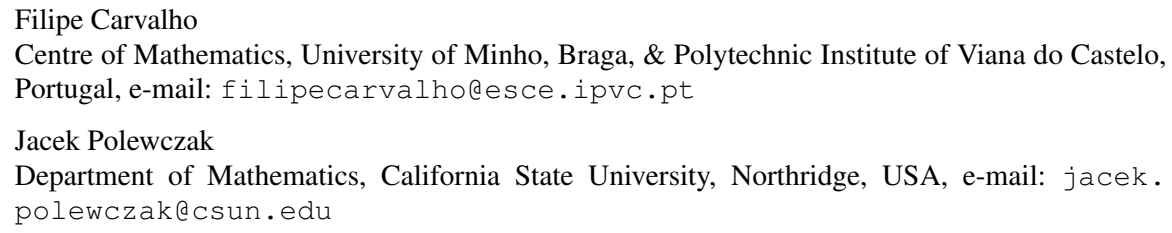


renormalized solutions for the SRS system, in the case that there is no mass transfer and no alteration of molecular diameters.

In the SRS kinetic theory, the molecules behave as if they were single mass points and the elastic and reactive collisions are assumed of hard-sphere type. Moreover, reactive collisions occur when the collision is sufficiently energetic, that is when the kinetic energy of the colliding molecules exceeds the activation energy of the chemical reaction. This theory incorporates some important aspects that renders the SRS kinetic model so interesting. For example, reactive and elastic collisions are treated in equal pair, contrary to those other models that consider the reactive terms as a small perturbation of the elastic ones (see, for example, [8], [9] and [10]). As a consequence, the SRS theory results to be appropriate to deal with processes considerably influenced by chemical reactions. Also, the dynamics of the reaction is fully integrated into the model, so that the detailed balance condition and the microscopic reversibility principle are built in the model. Additionally, when the chemical reactions are turned off, the SRS kinetic model reduces to the Boltzmann kinetic system of four species.

All these aspects motivate the authors to investigate some mathematical and physical problems for the SRS kinetic theory.

Continuing with the work presented in paper [2], we consider here a simplified version of the SRS model that accounts for a coloring process with probability $\alpha_{R}$ described by a reversible law of type $A_{1}+A_{2} \rightleftharpoons A_{3}+A_{4}$. Since this process does not modify the mass or chemical binding energy of the reactants, the reactive terms become straightforward and the corresponding SRS kinetic system results much simpler than the one of the general model considered in [2]. This allows to obtain a more tractable linearized SRS system and a better representation of the elastic and reactive kernels. The main purpose of the present paper is to use this representation of the kernels to prove the compactness of some of the elastic and reactive linearized operators, in particular, operators $\mathscr{Q}^{(1)}, \mathscr{Q}^{(2)}, \mathscr{T}^{(2)}$, and $\mathscr{R}^{(2)}$, in the notation of Section 6. On the other hand, additional techniques are required for the remaining operators, $\mathscr{Q}^{(3)}, \mathscr{T}^{(3)}$ and $\mathscr{R}^{(3)}$, and work is in progress to complete the proof of compactness for these operators. Once the compactness of these linearized operators is achieved, we plan to provide full spectral analysis of these operators, and this will be used to obtain expressions for the transport coefficients, the asymptotic behavior of the evolution operator associated with the linearized SRS system, and ultimately hydrodynamics limits for these systems.

The paper is organized as follows. In the next section, with reference to the considered coloring process, we describe the SRS kinetic model and state its consistency properties. Section 3 is devoted to the equilibrium solutions and trend to equilibrium. In Section 4 we present the linearized SRS system and state its main properties. In Section 5 we provide the explicit representation of the kernels of the linearized elastic and reactive operators and state some symmetry properties. Finally, in Section 6 we prove the compactness of two elastic and one reactive linearized operators. 


\section{SRS kinetic system}

With reference to the simple reacting spheres (SRS) model $[4,5,6]$, we consider a dilute gas mixture of four constituents, whose particles behave as if they were single mass points. Internal degrees of freedom for the gas particles, such as vibrational and rotational energies, are not taken into account. Particles undergo binary elastic collisions and reactive collisions, both of hard-sphere type. Reactive collisions obey the chemical law

$$
A+B \rightleftharpoons A^{*}+B^{*},
$$

where $A, B, A^{*}$ and $B^{*}$ indicate the constituents of the mixture. We use the indices $1,2,3,4$ for the constituents $A, B, A^{*}$ and $B^{*}$, respectively. Furthermore, $m_{i}, d_{i}$ and $E_{i}$ denote the molecular mass, the molecular diameter and the chemical binding energy of each $i$-constituent. In general, the chemical reaction results in a redistribution of masses among the constituents, in such a way that the law of mass conservation holds in the form

$$
m_{1}+m_{2}=m_{3}+m_{4}
$$

At the same time, the chemical reaction implies a rearrangement of energies (kinetic and binding) so that a conservation law of the total energy holds. The balance of chemical binding energy of products and reactants of the chemical reaction defines the reaction heat, namely $Q_{R}=E_{3}+E_{4}-E_{1}-E_{2}$. If $Q_{R}$ is positive, the reverse chemical reaction, $A^{*}+B^{*} \rightarrow A+B$, is exothermic.

In our paper [2], we described the SRS kinetic system for a general bimolecular chemical reaction. In particular, we introduced in detail the mathematical aspects of the SRS system and the relevant properties of the collisional operators for what concerns the mathematical and physical consistency of the model. Our main result in that paper is the representation of the SRS system linearized around the equilibrium and the explicit representation of the kernels of the reactive operators.

In the present paper we consider a simplified version of the SRS system, in which the reaction law (1) describes a coloring process with a fixed probability $\alpha_{R}$. We assume that

$$
m_{3}=m_{1}, m_{4}=m_{2}, \quad d_{3}=d_{1}, d_{4}=d_{2}, \quad E_{3}=E_{1}, E_{4}=E_{2} .
$$

When a hard-sphere $A$ collides with another hard-sphere $B$, there is a probability $\alpha_{R}$ that they change their colors in the process $A+B \rightarrow A^{*}+B^{*}$, without modifying their molecular masses or diameters, and without altering their chemical binding energies. The same probability rule is applied to the reverse reaction $A^{*}+B^{*}-$ $A+B$.

This dynamical model represents a simplified version of the SRS system that is appropriate to describe a coloring process. 


\subsection{Collisional dynamics}

An elastic collision between particles from constituents $i$ and $s$, with velocities $v$ and $w$, respectively, results in a change of velocities, say $(v, w) \rightarrow\left(v^{\prime}, w^{\prime}\right)$, with $i, s=$ $1, \ldots, 4$. Since the linear momentum and kinetic energy of the colliding particles are conserved, we have

$$
\begin{gathered}
m_{i} v+m_{s} w=m_{i} v^{\prime}+m_{s} w^{\prime} \\
m_{i} v^{2}+m_{s} w^{2}=m_{i} v^{2}+m_{s} w^{\prime 2}
\end{gathered}
$$

We consider elastic cross sections of hard-spheres type, given by

$$
\sigma_{i s}^{2}=\frac{1}{4}\left(d_{i}+d_{s}\right)^{2}
$$

Conditions (3) and (4), imply that the elastic post-collisional velocities are given by

$$
v^{\prime}=v-2 \frac{\mu_{i s}}{m_{i}}\langle\varepsilon, v-w\rangle \varepsilon \quad \text { and } \quad w^{\prime}=w+2 \frac{\mu_{i s}}{m_{s}}\langle\varepsilon, v-w\rangle \varepsilon
$$

where $\varepsilon$ is a unit vector along the line passing through the centres of the colliding particles at the moment of the impact, and $\mu_{i s}$ is the reduced mass of the colliding particles,

$$
\varepsilon \in \mathbb{S}_{+}^{2}, \text { with } \mathbb{S}_{+}^{2}=\left\{\varepsilon \in \mathbb{R}^{3}:\|\varepsilon\|=1,\langle\varepsilon, v-w\rangle>0\right\}, \text { and } \mu_{i s}=\frac{m_{i} m_{s}}{m_{i}+m_{s}} .
$$

On the other hand, a reactive collision in our model is a coloring event in which the participating molecules only change, besides their velocities, their color. Thus the reactive collision between particles from constituents $i$ and $j$ with velocities $v$ and $w$, respectively, results in a transition into constituents $k$ and $l$ with different colors from $i$ and $j$, and a consequent change of velocities to $v^{\odot}$ and $w^{\odot}$. The indices $(i, j, k, l)$ can take the values $(1,2,3,4),(2,1,4,3),(3,4,1,2)$ and $(4,3,2,1)$. The conservation laws of linear momentum and total energy of the colliding particles are represented by

$$
\begin{aligned}
m_{1} v+m_{2} w & =m_{3} v^{\odot}+m_{4} w^{\odot} \\
m_{1} v^{2}+m_{2} w^{2} & =m_{3}\left(v^{\odot}\right)^{2}+m_{4}\left(w^{\odot}\right)^{2},
\end{aligned}
$$

where the molecular masses satisfy conditions (2). The chemical reactive cross sections associated to the coloring process are given by

$$
\sigma_{12}^{* 2}=\alpha_{R} \sigma_{12}^{2} \quad \text { and } \quad \sigma_{34}^{* 2}=\alpha_{R} \sigma_{34}^{2}
$$

for the direct and inverse reaction, respectively. The post-collisional velocities associated to the direct reaction $A+B \rightarrow A^{*}+B^{*}$, for the constituents $k$ and $l$, are given by 


$$
v^{\odot}=v-2 \frac{\mu_{i j}}{m_{k}}\langle\varepsilon, v-w\rangle \varepsilon \quad \text { and } \quad w^{\odot}=w+2 \frac{\mu_{i j}}{m_{l}} \varepsilon\langle\varepsilon, v-w\rangle .
$$

where $(i, j, k, l) \in\{(1,2,3,4),(2,1,4,3),(3,4,1,2),(4,3,2,1)\}$. The post-collisional velocities associated to the backward reaction $A^{*}+B^{*} \rightarrow A+B$, for the constituents $i$ and $j$, are given by the same expressions (9), thanks to the assumptions (2) on the molecular masses.

\subsection{Kinetic system}

Within the kinetic theory for rarefied gases, the state of the mixture is described by the one-particle distribution functions $f_{i}(t, x, v), i=1,2,3,4$, such that $f_{i}(t, x, v)$ represents the probability density of finding, at time $t$, a particle of the $A_{i}$-constituent occupying the position $x$ with velocity $v$. Here, the time-space evolution of the functions $f_{i}(t, x, v)$ is given by the SRS kinetic system formulated for the coloring problem, in the form

$$
\frac{\partial f_{i}}{\partial t}+v \cdot \nabla_{x} f_{i}=J_{i}^{E}+J_{i}^{R}, \quad i=1, \ldots, 4,
$$

where $J_{i}^{E}$ is the elastic collisional operator, given by

$$
\begin{aligned}
J_{i}^{E}= & \sum_{s=1}^{4} \sigma_{i s}^{2} \int_{\mathbb{R}^{3}} \int_{\mathbb{S}_{+}^{2}}\left[f_{i}\left(t, x, v^{\prime}\right) f_{s}\left(t, x, w^{\prime}\right)-f_{i}(t, x, v) f_{s}(t, x, w)\right]\langle\varepsilon, v-w\rangle d \varepsilon d w \\
& -\alpha_{R} \sigma_{i j}^{2} \int_{\mathbb{R}^{3}} \int_{\mathbb{S}_{+}^{2}}\left[f_{i}\left(t, x, v^{\prime}\right) f_{j}\left(t, x, w^{\prime}\right)-f_{i}(t, x, v) f_{j}(t, x, w)\right]\langle\varepsilon, v-w\rangle d \varepsilon d w
\end{aligned}
$$

with $(i, j) \in\{(1,2),(2,1),(3,4),(4,3)\}$, and $J_{i}^{R}$ is the reactive collisional operator, given by

$$
J_{i}^{R}=\alpha_{R} \sigma_{i j}^{2} \int_{\mathbb{R}^{3}} \int_{\mathbb{S}_{+}^{2}}\left[f_{k}\left(t, x, v^{\odot}\right) f_{l}\left(t, x, w^{\odot}\right)-f_{i}(t, x, v) f_{j}(t, x, w)\right]\langle\varepsilon, v-w\rangle d \varepsilon d w,
$$

with $(i, j, k, l) \in\{(1,2,3,4),(2,1,4,3),(3,4,1,2),(4,3,2,1)\}$. Velocities $v^{\prime}, w^{\prime}$ and $v^{\odot}, w^{\odot}$ are given by Eqs. (5) and (9), respectively. Moreover, the second term in the expression (11) of $J_{i}^{E}$, with $0 \leq \alpha_{R} \leq 1$ in front of it, singles out those collisions that result in a coloring process and prevent double counting of the events in the elastic collisional integrals.

The collisional operators $J_{i}^{E}$ and $J_{i}^{R}$ satisfy some mathematical properties, which assure the physical consistency of the model, at least from the formal point of view.

Proposition 1 The collisional operators are such that

(a) $\int_{\mathbb{R}^{3}} J_{i}^{E} d v=0, \quad i=1,2,3,4$; 
(b) $\int_{\mathbb{R}^{3}} J_{1}^{R} d v=\int_{\mathbb{R}^{3}} J_{2}^{R} d v=-\int_{\mathbb{R}^{3}} J_{3}^{R} d v=-\int_{\mathbb{R}^{3}} J_{4}^{R} d v$;

(c) $\sum_{i=1}^{4} \int_{\mathbb{R}^{3}} \psi_{i}\left(J_{i}^{E}+J_{i}^{R}\right) d v=0$, where $\psi=\left(\psi_{1}, \psi_{2}, \psi_{3}, \psi_{4}\right)$ is alternatively given by $\psi=(1,0,1,0), \psi=(1,0,0,1), \psi=(0,1,1,0)$, or by $\psi_{i}=m_{i} v_{1}, \psi_{i}=m_{i} v_{2}$, $\psi_{i}=m_{i} v_{3}$, with $v_{1}, v_{2}, v_{3}$ being the spatial components of the velocity $v$, and $\psi_{i}=\frac{1}{2} m_{i} v^{2}$.

Property $(a)$ assures that elastic collisions do not modify the number of particles of each color.

Property $(b)$ states the correct exchange rates of all constituents, in agreement with the chemical law (1) describing the coloring process.

Property $(c)$ implies seven independent macroscopic conservation laws, for the total number of particles of the reactant-product pairs of the form $A_{1}-A_{3}, A_{1}-A_{4}$ and $A_{2}-$ $A_{3}$, as well as for the three momentum components and kinetic energy of the whole mixture. Proper linear combinations of the former three functions $\psi$ in $(c)$ reproduce conservation of the particle number density and of total mass of the mixture. Note that, in contrast to a non-reactive mixture, there is no mass conservation for the individual constituents in our simple model.

The conservation laws involve macroscopic quantities defined, as usual, as appropriate velocity moments of the distribution functions $f_{i}(t, x, v)$. Paper [2] includes a rather complete definition of the macroscopic variables for the more general SRS system with bimolecular chemical reaction. The paper also includes the set of balance equations and conservation laws of the system. Here, for sake of brevity, we omit the analysis of the macroscopic framework of the model.

\section{Equilibrium solutions and H-Theorem}

Definition 1 The equilibrium solutions of system (10), (11) and (12) are distribution functions $f_{i}(t, x, v)$ satisfying

$$
J_{i}^{E}+J_{i}^{R}=0, \quad i=1, \ldots, 4 .
$$

The above definition of equilibrium solutions is motivated by the following result:

Proposition 2 For $n_{i}(t, x), u(t, x)$, and $T(t, x)$ with $f_{i}(t, x, v) \geq 0$, the following statements are equivalent:

$$
\text { 1. } M_{i}=n_{i}\left(\frac{m_{i}}{2 \pi k T}\right)^{3 / 2} \exp \left(-\frac{m_{i}(v-u)^{2}}{2 k T}\right), i=1, \ldots, 4 \text {, and } n_{1} n_{2}=n_{3} n_{4} \text {; }
$$


2. $J_{i}^{E}\left(\left\{M_{i}\right\}\right)=0$ and $J_{i}^{R}\left(\left\{M_{i}\right\}\right)=0, \quad i=1, \ldots, 4$;

3. $\sum_{i=1}^{4} \int_{\mathbb{R}^{3}}\left[J_{i}^{E}\left(\left\{M_{i}\right\}\right)+J_{i}^{R}\left(\left\{M_{i}\right\}\right)\right] \log \left(M_{i} / \mu_{i j}\right) d v=0$.

The notations $J_{i}^{E}\left(\left\{M_{i}\right\}\right)$ and $J_{i}^{R}\left(\left\{M_{i}\right\}\right)$ signify the fact that for $i=1, \ldots, 4$, the collisional operators depend on the set one-particle distribution functions, $M_{1}, M_{2}, M_{3}$, and $M_{4}$. Also $\mu_{i j}=m_{i} m_{j} /\left(m_{i}+m_{j}\right)$ with $(i, j)=(1,2),(2,1),(3,4),(4,3)$, and

$$
\begin{aligned}
n_{i} & =\int_{\mathbb{R}^{3}} f_{i}(t, x, v) d v, \quad i=1, \ldots, 4, \\
u & =\sum_{i=1}^{4} \int_{\mathbb{R}^{3}} m_{i} v f_{i}(t, x, v) d v / \sum_{i=1}^{4} \int_{\mathbb{R}^{3}} m_{i} f_{i}(t, x, v) d v, \\
T & =\frac{1}{3 k} \sum_{i=1}^{4} \int_{\mathbb{R}^{3}} m_{i}(v-u)^{2} f_{i}(t, x, v) d v / \sum_{i=1}^{4} n_{i},
\end{aligned}
$$

where $k$ is the Boltzmann constant.

Equation $n_{1} n_{2}=n_{3} n_{4}$ appearing in item 1. of Proposition 2 represents the mass action law for our coloring system. The proof of Proposition 2 follows the same line of arguments as the proof of Proposition 3.2 in [7]. See also the discussion in Refs. $[2,11]$.

The Maxwellian distribution functions satisfy the properties stated in the next two lemmas, which will be used in the next section.

Lemma 1. The Maxwellian distribution functions $M_{i}$ appearing in Proposition 2 with the number densities $n_{i}$ are such that

$$
M_{i}\left(t, x, v^{\prime}\right) M_{s}\left(t, x, w^{\prime}\right)=M_{i}(t, x, v) M_{s}(t, x, w), \quad i, s=1,2,3,4,
$$

where $v$ and $w$ are pre-collisional velocities of species $A_{i}$ and $A_{s}$, respectively, and $v^{\prime}$ and $w^{\prime}$ are the corresponding elastic post-collisional velocities given by (5).

Lemma 2. The Maxwellian distribution functions $M_{i}$ with number densities $n_{i}$ constrained by the mass action law are such that

$$
M_{k}\left(t, x, v^{\odot}\right) M_{l}\left(t, x, w^{\odot}\right)=M_{i}(t, x, v) M_{j}(t, x, w),
$$

where $(i, j, k, l) \in\{(1,2,3,4),(2,1,4,3),(3,4,1,2),(4,3,2,1)\}, v$ and $w$ are the precollisional velocities of constituents $A_{i}$ and $A_{j}$, respectively, and $v^{\odot}$ and $w^{\odot}$ are the corresponding reactive post-collisional velocities of constituents $A_{k}$ and $A_{l}$, respectively, given by (9).

Lemma 1 follows from the energy conservation of elastic events (3b). and Lemma 2 follows from the law of mass action and from the energy conservation of reactive events $(7 b)$. 
The trend to equilibrium of the reactive mixture and the macroscopic irreversibility of the coloring process are assured by an $\mathscr{H}$-theorem. In fact, for a nonnegative smooth solution $f_{i}(t, x, v)$ of our kinetic system (10), (11) and (12), the convex function $H(t)$ defined by

$$
H(t)=\sum_{i=1}^{4} \int_{\Omega} \int_{\mathbb{R}^{3}} f_{i}(t, x, v) \log \left[f_{i}(t, x, v) / \mu_{i j}\right] d v d x,
$$

is a Liapunov functional (see for example, [7]). Here, $\mu_{i j}$ is the reduced mass defined in Proposition 2, and $(i, j)=(1,2),(2,1),(3,4),(4,3)$. Moreover, $H(t)$ attains its minimum when the velocity distribution functions are Maxwellians given in Proposition 2. Here, $\Omega$ is the physical evolution domain of the mixture. We assume that $\Omega$ can be either the whole space $\mathbb{R}^{3}$ or a box with boundary conditions of periodic type or boundary conditions of specular reflection at the walls.

\section{Linearized SRS kinetic system}

We assume that the evolution regime of the gas mixture corresponds to a small deviation of the thermodynamical equilibrium. We then expand the distribution function $f_{i}(t, x, v)$ around the Maxwellian $M_{i}(t, x, v)$ with zero drift velocity $u=0$ and densities satisfying the mass action law. We introduce a new unknown function $h_{i}(t, x, v)$ connected to $f_{i}(t, x, v)$ through the expansions

$$
f_{i}(t, x, v)=M_{i}(t, x, v)+M_{i}^{1 / 2}(t, x, v) h_{i}(t, x, v), \quad i=1, \ldots, 4 .
$$

For each $i=1, \ldots, 4$, the term $M_{i}^{1 / 2} h_{i}$ represents the deviation of the distribution function $f_{i}$ from the equilibrium. The factors $M_{i}^{1 / 2}$ in front of $h_{i}, i=1, \ldots, 4$, make the linearized operator symmetric in $L^{2}$ space without a need to introduce additional weigh functions, see [13]. Inserting expansions (18) into Eqs. (10-12), and using the properties (15) and (16), we obtain the linearized SRS kinetic system, as stated in the next proposition. For sake of brevity, we use the notations $\underline{h}=\left(h_{1}, h_{2}, h_{3}, h_{4}\right)^{\mathrm{T}}$, $h_{i}(v)$ instead of $h_{i}(t, x, v)$, and $\widehat{h}_{i}=M_{i}^{1 / 2} h_{i}$.

Proposition 3 The linearized SRS kinetic system for our model is given by

$$
\frac{\partial \widehat{h}_{i}}{\partial t}+v \cdot \nabla_{x} \widehat{h}_{i}=\mathscr{L}_{i}^{E}(\underline{\widehat{h}})+\mathscr{L}_{i}^{R}(\underline{\widehat{h}}) \equiv \mathscr{L}_{i}(\underline{\widehat{h}}), \quad i=1, \ldots, 4,
$$

with 


$$
\begin{aligned}
\mathscr{L}_{i}^{E}(\underline{\hat{h}})=\sum_{s=1}^{4} \sigma_{i s}^{2} \int_{\mathbb{R}^{3}} \int_{\mathbb{S}_{+}^{2}}\left[M_{s}(w) M_{i}^{1 / 2}(v) M_{i}^{-1 / 2}\left(v^{\prime}\right) \widehat{h}_{i}\left(v^{\prime}\right)\right. \\
+M_{s}(w) M_{i}^{1 / 2}(v) M_{s}^{-1 / 2}\left(w^{\prime}\right) \widehat{h}_{s}\left(w^{\prime}\right)-M_{s}(w) \widehat{h}_{i}(v) \\
\left.-M_{s}^{1 / 2}(w) M_{i}^{1 / 2}(v) \widehat{h}_{s}(w)\right]\langle\varepsilon, v-w\rangle d \varepsilon d w \\
-\alpha_{R} \sigma_{i j}^{2} \int_{\mathbb{R}^{3}} \int_{\mathbb{S}_{+}^{2}}\left[M_{j}(w) M_{i}^{1 / 2}(v) M_{i}^{-1 / 2}\left(v^{\prime}\right) \widehat{h}_{i}\left(v^{\prime}\right)\right. \\
+M_{j}(w) M_{i}^{1 / 2}(v) M_{j}^{-1 / 2}\left(w^{\prime}\right) \widehat{h}_{j}\left(w^{\prime}\right)-M_{j}(w) \widehat{h}_{i}(v) \\
\left.-M_{j}^{1 / 2}(w) M_{i}^{1 / 2}(v) \widehat{h}_{j}(w)\right]\langle\varepsilon, v-w\rangle d \varepsilon d w \\
\mathscr{L}_{i}^{R}(\underline{\hat{h}})=\alpha_{R} \sigma_{i j}^{2} \int_{\mathbb{R}^{3}} \int_{\mathbb{S}_{+}^{2}}\left[M_{j}(w) M_{i}^{1 / 2}(v) M_{k}^{-1 / 2}\left(v^{\odot}\right) \widehat{h}_{k}\left(v^{\odot}\right)\right. \\
+M_{j}(w) M_{i}^{1 / 2}(v) M_{l}^{-1 / 2}\left(w^{\odot}\right) \widehat{h}_{l}\left(w^{\odot}\right)-M_{j}(w) \widehat{h}_{i}(v) \\
\left.-M_{j}^{1 / 2}(w) M_{i}^{1 / 2}(v) \widehat{h}_{j}(w)\right]\langle\varepsilon, v-w\rangle d \varepsilon d w
\end{aligned}
$$

where $(i, j, k, l) \in\{(1,2,3,4),(2,1,4,3),(3,4,1,2),(4,3,2,1)\}$.

We introduce the linearized collision operator $\mathscr{L}$ with components $\mathscr{L}_{i}=\mathscr{L}_{i}^{E}+$ $\mathscr{L}_{i}^{R}, i=1,2,3,4$, where $\mathscr{L}_{i}^{E}$ and $\mathscr{L}_{i}^{R}$ are defined above. Such operator possesses fundamental properties that are stated below. At this end, we consider the Hilbert space $Y=L^{2}\left(\mathbb{R}^{3}\right) \times L^{2}\left(\mathbb{R}^{3}\right) \times L^{2}\left(\mathbb{R}^{3}\right) \times L^{2}\left(\mathbb{R}^{3}\right)$ endowed with the inner product defined by

$$
\langle\underline{F}, \underline{G}\rangle=\sum_{i=1}^{4} \int_{\mathbb{R}^{3}} F_{i}(v) G_{i}(v) d v,
$$

for $\underline{F}=\left(F_{1}, F_{2}, F_{3}, f_{4}\right) \in Y$ and $\underline{G}=\left(G_{1}, G_{2}, G_{3}, G_{4}\right) \in Y$, with $F_{i}, G_{i}(i=1,2,3,4)$ real-valued functions. Then we have the following result.

Proposition 4 The linearized collisional operator $\mathscr{L}$ is symmetric and non-positive semi-definite, that is

(a) $\langle\mathscr{L}(\underline{F}), \underline{G}\rangle=\langle\underline{F}, \mathscr{L}(\underline{G})\rangle$, for all $\underline{F}, \underline{G} \in Y$;

(b) $\langle\mathscr{L}(\underline{F}), \underline{F}\rangle \leq 0$, for all $\underline{F} \in Y$. Additionally, $\langle\mathscr{L}(\underline{F}), \underline{F}\rangle=0$ if and only if $\underline{F}$ is a collisional invariant weighted by $\underline{M}^{1 / 2}=\left(M_{1}^{1 / 2}, M_{2}^{1 / 2}, M_{3}^{1 / 2}, M_{4}^{1 / 2}\right)$, that is the null space of the operator $\mathscr{L}$ is 7-dimensional and is spanned by the collisional invariants (weighted by $\underline{M}^{1 / 2}$ ) associated to the conservation laws of the partial number densities $n_{1}+n_{3}, n_{1}+n_{4}, n_{2}+n_{3}$, momentum components and kinetic energy of the mixture. 


\section{Kernels of the linearized operators}

In the case of a gas mixture, even if without chemical reaction, the representation of the kernels is much more involved than in the case of a single inert gas. In fact, the complexity of the collisional terms increase significantly when collisions between particles of different constituents and different molecular masses are considered, especially when chemical reactions are also allowed. The computation of the kernels of the linearized collisional operators requires much more work and therefore the explicit representation of the kernels becomes, in our opinion, a very difficult task.

In the last years, we have been interested in this topic and some results have been obtained in this direction. In particular, the explicit representation of the kernels of the SRS linearized operators, both elastic and reactive, is presented in [11], for the general case of a quaternary mixture with reversible bimolecular reaction and arbitrary molecular masses. The expressions are, in general, quite long and rather complicated, because some of the transformations used to compute the kernels do not retain the symmetry of the integral operators. A better representation has been obtained in paper [2], for a similar chemically reactive mixture but considering that the constituents have equal molecular masses. The expressions are more tractable but those of the reactive operators are still intricate.

For the simplified version of the SRS model considered in the present paper, we were able to obtain a rather simple representation of the kernels. In particular, the expressions of both elastic and reactive kernels keep the symmetry property of the corresponding integral operators. In this section, we give the kernels of the operators and briefly explain the computations. We also state some symmetry properties of the kernels useful in the next section 6 .

\subsection{Explicit representation of the kernels}

In view of the explicit computations, we first work on the expression of the linearized operator $\mathscr{L}_{i}$ by manipulating expressions (20) and (21) of $\mathscr{L}_{i}^{E}$ and $\mathscr{L}_{i}^{R}$. The last two contributions of $\mathscr{L}_{i}^{E}$ and $\mathscr{L}_{i}^{R}$ are equal with opposite signs and thus they cancel out. Then, using the energy conservation (3b) of elastic events we modify those contributions appearing in the expression of $\mathscr{L}_{i}^{E}$ with the perturbation function $\widehat{h}$ evaluated in the post collisional velocities $v^{\prime}$ and $w^{\prime}$.

Analogously, we use the energy conservation (7b) of the reactive events and the mass action law to work on those contributions in $\mathscr{L}_{i}^{R}$ with $\widehat{h}$ evaluated in the post collisional velocities $v^{\odot}$ and $w^{\odot}$. Using the same notations $\mathscr{L}_{i}^{E}$ and $\mathscr{L}_{i}^{R}$ for the elastic and reactive contributions in the resulting expression of $\mathscr{L}_{i}$, we obtain 


$$
\begin{gathered}
\mathscr{L}_{i}^{E}(\underline{\hat{h}})=\sum_{s=1}^{4} \sigma_{i s}^{2} \int_{\mathbb{R}^{3}} \int_{\mathbb{S}_{+}^{2}}\left[M_{s}^{1 / 2}(w) M_{s}^{1 / 2}\left(w^{\prime}\right) \widehat{h}_{i}\left(v^{\prime}\right)+M_{s}^{1 / 2}(w) M_{i}{ }^{1 / 2}\left(v^{\prime}\right) \widehat{h}_{s}\left(w^{\prime}\right)\right. \\
\left.-M_{s}(w) \widehat{h}_{i}(v)-M_{s}^{1 / 2}(w) M_{i}^{1 / 2}(v) \widehat{h}_{s}(w)\right]\langle\varepsilon, v-w\rangle d \varepsilon d w \\
-\alpha_{R} \sigma_{i j}^{2} \int_{\mathbb{R}^{3}} \int_{\mathbb{S}_{+}^{2}}\left[M_{j}^{1 / 2}(w) M_{j}^{1 / 2}\left(w^{\prime}\right) \widehat{h}_{i}\left(v^{\prime}\right)\right. \\
\left.+M_{j}^{1 / 2}(w) M_{i}^{1 / 2}\left(v^{\prime}\right) \widehat{h}_{j}\left(w^{\prime}\right)\right]\langle\varepsilon, v-w\rangle d \varepsilon d w, \\
\mathscr{L}_{i}^{R}(\underline{\hat{h}})=\alpha_{R} \sigma_{i j}^{2} \int_{\mathbb{R}^{3}} \int_{\mathbb{S}_{+}^{2}}\left[M_{j}^{1 / 2}(w) M_{l}^{1 / 2}\left(w^{\odot}\right) \widehat{h}_{k}\left(v^{\odot}\right)\right. \\
\left.+M_{j}^{1 / 2}(w) M_{k}^{1 / 2}\left(v^{\odot}\right) \widehat{h}_{l}\left(w^{\odot}\right)\right]\langle\varepsilon, v-w\rangle d \varepsilon d w,
\end{gathered}
$$

where $(i, j, k, l) \in\{(1,2,3,4),(2,1,4,3),(3,4,1,2),(4,3,2,1)\}$.

\subsubsection{Kernels of the elastic operators}

We split the elastic linearized operator $\mathscr{L}_{i}^{E}$ given by (23) in several contributions, as follows

$$
\mathscr{L}_{i}^{E}(\underline{h})=-v_{i}(v) \widehat{h}_{i}(v)-\mathscr{Q}_{i}^{(1)}(\underline{h})+\mathscr{Q}_{i}^{(2)}(\underline{h})+\mathscr{Q}_{i}^{(3)}(\underline{h})-\mathscr{T}_{i}^{(2)}(\underline{h})-\mathscr{T}_{i}^{(3)}(\underline{h}),
$$

with

$$
\begin{aligned}
& v_{i}(v)=\sum_{s=1}^{4} \sigma_{i s}^{2} \int_{\mathbb{R}^{3}} \int_{\mathbb{S}_{+}^{2}} M_{s}(w)\langle\varepsilon, v-w\rangle d \varepsilon d w \\
& \mathscr{Q}_{i}^{(1)}(\widehat{\widehat{h}})=\sum_{s=1}^{4} \sigma_{i s}^{2} \int_{\mathbb{R}^{3}} \int_{\mathbb{S}_{+}^{2}} M_{i}^{1 / 2}(v) M_{s}^{1 / 2}(w) \widehat{h}_{s}(w)\langle\varepsilon, v-w\rangle d \varepsilon d w \\
& \mathscr{Q}_{i}^{(2)}(\underline{\hat{h}})=\sum_{s=1}^{4} \sigma_{i s}^{2} \int_{\mathbb{R}^{3}} \int_{\mathbb{S}_{+}^{2}} M_{s}^{1 / 2}(w) M_{s}^{1 / 2}\left(w^{\prime}\right) \widehat{h}_{i}\left(v^{\prime}\right)\langle\varepsilon, v-w\rangle d \varepsilon d w \\
& \mathscr{Q}_{i}^{(3)}(\underline{\hat{h}})=\sum_{s=1}^{4} \sigma_{i s}^{2} \int_{\mathbb{R}^{3}} \int_{\mathbb{S}_{+}^{2}} M_{s}^{1 / 2}(w) M_{i}^{1 / 2}\left(v^{\prime}\right) \widehat{h}_{s}\left(w^{\prime}\right)\langle\varepsilon, v-w\rangle d \varepsilon d w \\
& \mathscr{T}_{i}^{(2)}(\underline{\hat{h}})=\alpha_{R} \sigma_{i j}^{2} \int_{\mathbb{R}^{3}} \int_{\mathbb{S}_{+}^{2}} M_{j}^{1 / 2}(w) M_{j}^{1 / 2}\left(w^{\prime}\right) \widehat{h}_{i}\left(v^{\prime}\right)\langle\varepsilon, v-w\rangle d \varepsilon d w \\
& \mathscr{T}_{i}^{(3)}(\underline{\widehat{h}})=\alpha_{R} \sigma_{i j}^{2} \int_{\mathbb{R}^{3}} \int_{\mathbb{S}_{+}^{2}} M_{j}^{1 / 2}(w) M_{i}^{1 / 2}\left(v^{\prime}\right) \widehat{h}_{j}\left(w^{\prime}\right)\langle\varepsilon, v-w\rangle d \varepsilon d w
\end{aligned}
$$

where $v_{i}(v)$ represents a generalized collision frequency.

We work separately on each operator. It is easy to extract the kernel of each operator $\mathscr{Q}_{i}^{(1)}$ and $\mathscr{Q}_{i}^{(2)}$. We obtain 


$$
\begin{aligned}
& \mathscr{Q}_{i}^{(1)}(\underline{\hat{h}})=\sum_{s=1}^{4} \int_{\mathbb{R}^{3}} K_{i s}^{(1)}(v, w) \widehat{h}_{s}(w) d w, \\
& \mathscr{Q}_{i}^{(2)}(\underline{\underline{h}})=\sum_{s=1}^{4} \int_{\mathbb{R}^{3}} K_{i s}^{(2)}(v, w) \widehat{h}_{i}(w) d w,
\end{aligned}
$$

with the kernels $K_{i s}^{(1)}$ and $K_{i s}^{(2)}(i, s=1, \ldots, 4)$ given by

$$
\begin{aligned}
& K_{i s}^{(1)}(v, w)=\pi \sigma_{i s}^{2}\|v-w\| \sqrt{n_{i} n_{s}}\left(\frac{m_{i} m_{s}}{(2 \pi k T)^{2}}\right)^{3 / 4} \exp \left(-\frac{m_{i} v^{2}+m_{s} w^{2}}{4 k T}\right), \\
& K_{i s}^{(2)}(v, w)= \sigma_{i s}^{2} n_{s}\left(\frac{m_{s}}{2 \pi k T}\right)^{1 / 2}\left(\frac{m_{i}}{2 \mu_{i s}}\right)^{2} \frac{1}{\|v-w\|} \\
& \times \exp \left[-\frac{m_{s}}{8 k T} \frac{\left(v^{2}-w^{2}\right)^{2}}{\|v-w\|^{2}}-\frac{m_{s}}{8 k T}\left(\frac{m_{i}}{m_{s}}\right)^{2}(v-w)^{2}\right] .
\end{aligned}
$$

The computation of the kernel of $\mathscr{Q}_{i}^{(3)}$ requires more work. It results convenient to split the operator $\mathscr{Q}_{i}^{(3)}$ as a summation according to the molecular masses $m_{s}$ and $m_{i}$. Thus, we introduce the following sets of indices

$$
\begin{gathered}
I=\{1,2,3,4\}, \\
I^{0}=\left\{s \in I: m_{s}=m_{i}\right\}, I^{+}=\left\{s \in I: m_{s}>m_{i}\right\}, I^{-}=\left\{s \in I: m_{s}<m_{i}\right\},
\end{gathered}
$$

and split the operator $\mathscr{Q}_{i}^{(3)}$ into three contributions, namely

$$
\mathscr{Q}_{i}^{(3)}(\underline{\widehat{h}})=\mathscr{Q}_{i}^{\left(3^{0}\right)}(\underline{\widehat{h}})+\mathscr{Q}_{i}^{\left(3^{+}\right)}(\underline{\widehat{h}})+\mathscr{Q}_{i}^{\left(3^{-}\right)}(\underline{\widehat{h}}),
$$

where

$$
\begin{aligned}
& \mathscr{Q}_{i}^{\left(3^{0}\right)}(\underline{\hat{h}})=\sum_{s \in I^{0}} \int_{\mathbb{R}^{3}} K_{i s}^{\left(3^{0}\right)}(v, w) \widehat{h}_{s}(w) d w, \\
& \mathscr{Q}_{i}^{\left(3^{+}\right)}(\underline{\widehat{h}})=\sum_{s \in I^{+}} \int_{\mathbb{R}^{3}} K_{i s}^{\left(3^{+}\right)}(v, w) \widehat{h}_{s}(w) d w, \\
& \mathscr{Q}_{i}^{\left(3^{-}\right)}(\underline{\widehat{h}})=\sum_{s \in I^{-}} \int_{\mathbb{R}^{3}} K_{i s}^{\left(3^{-}\right)}(v, w) \widehat{h}_{s}(w) d w .
\end{aligned}
$$

Then we obtain the kernels of the operators (37-39) in the form 


$$
\begin{aligned}
K_{i s}^{\left(3^{0}\right)}(v, w)= & \sigma_{i s}^{2}\left(n_{i} n_{s}\right)^{1 / 2}\left(\frac{m_{i}}{2 \pi k T}\right)^{1 / 2} \frac{1}{\|v-w\|} \\
& \times \exp \left[-\frac{m_{i}}{8 k T} \frac{\left(v^{2}-w^{2}\right)^{2}}{\|v-w\|^{2}}-\frac{m_{i}}{8 k T}(v-w)^{2}\right], i=1,2,3,4, s \in I^{0} \\
K_{i s}^{\left(3^{+}\right)}(v, w)= & \frac{2 \pi \sigma_{i s}^{2}}{\left(\frac{m_{s}-m_{i}}{m_{s}+m_{i}}\right)^{2}}\left(n_{i} n_{s}\right)^{1 / 2}\left(\frac{m_{i} m_{s}}{(2 \pi k T)^{2}}\right)^{3 / 4}\|v-w\| \\
& \times \exp \left[-\frac{1}{4 k T} \frac{\left(m_{s} w-m_{i} v\right)^{2}}{m_{i}+m_{s}}\right] \\
& \times \int_{0}^{\pi / 2} \cos ^{2} \theta \exp \left[-\frac{\mu_{i s}}{k T}\left(\left(\frac{\|v+w\|}{2}-\frac{\|v-w\| \cos \theta}{\frac{m_{s}-m_{i}}{m_{s}+m_{i}}}\right)^{2}\right.\right. \\
& \left.\left.+\frac{\|v-w\|\|v+w\| \cos \theta\left(1-\cos \theta^{\star}\right)}{\frac{m_{s}-m_{i}}{m_{s}+m_{i}}}\right)\right] d \theta, i=1,2,3,4, s \in I^{+}, \\
K_{i s}^{\left(3^{-}\right)}(v, w)= & \frac{2 \pi \sigma_{i s}^{2}}{\left(\frac{m_{s}-m_{i}}{m_{s}+m_{i}}\right)^{2}\left(n_{i} n_{s}\right)^{1 / 2}\left(\frac{m_{i} m_{s}}{(2 \pi k T)^{2}}\right)^{3 / 4}\|v-w\|} \\
& \times \exp \left[-\frac{1}{4 k T} \frac{\left(m_{s} w-m_{i} v\right)^{2}}{m_{i}+m_{s}}\right] d \theta, i=1,2,3,4, s \in I^{-} . \\
& \times \int_{\pi / 2}^{\pi} \cos ^{2} \theta \exp \left[-\frac{\mu_{i s}}{k T}\left(\left(\frac{\|v+w\|}{2}-\frac{\|v-w\| \cos \theta}{\frac{m_{s}-m_{i}}{m_{s}+m_{i}}}\right)^{2}\right.\right. \\
& \left.\frac{\|v-w\|\|v+w\| \cos \theta\left(1-\cos \theta^{\star}\right)}{m_{s}+m_{i}}\right) d \theta
\end{aligned}
$$

In the above equations (41) and (42), $\theta^{\star}$ is the angle defined by the vectors $v+w$ and $\varepsilon$. Observe that, in (41), we have $m_{s}>m_{i}$ because $s \in I^{+}$, whereas in (42), we have $m_{s}<m_{i}$, because $s \in I^{-}$.

Now we proceed with the operators $\mathscr{T}_{i}^{(\alpha)}$. We obtain

$$
\begin{aligned}
& \mathscr{T}_{i}^{(2)}(\underline{\widehat{h}})=\int_{\mathbb{R}^{3}} N_{i j}^{(2)}(v, w) \widehat{h}_{i}(w) d w, \\
& \mathscr{T}_{i}^{(3)}(\underline{\widehat{h}})=\int_{\mathbb{R}^{3}} N_{i j}^{(3)}(v, w) \widehat{h}_{j}(w) d w,
\end{aligned}
$$

and the computations are similar to those developed for $\mathscr{Q}_{i}^{(2)}$ and $\mathscr{Q}_{i}^{(3)}$. For sake of brevity, we omit their expressions here. 


\subsubsection{Kernels of the reactive operators}

The calculation of the reactive kernels are similar. We start by splitting the linearized reactive collisional operator (24) as follows

$$
\widehat{\mathscr{L}}_{i}^{R}(\underline{\hat{h}})=\mathscr{R}_{i}^{(2)}(\underline{\widehat{h}})+\mathscr{R}_{i}^{(3)}(\underline{\widehat{h}})
$$

with

$$
\begin{aligned}
& \mathscr{R}_{i}^{(2)}(\underline{\hat{h}})=\alpha_{R} \sigma_{i j}^{2} \int_{\mathbb{R}^{3}} \int_{\mathbb{S}_{+}^{2}} M_{j}^{1 / 2}(w) M_{l}^{1 / 2}\left(w^{\odot}\right) \widehat{h}_{k}\left(v^{\odot}\right)\langle\varepsilon, v-w\rangle d \varepsilon d w, \\
& \mathscr{R}_{i}^{(3)}(\underline{\widehat{h}})=\alpha_{R} \sigma_{i j}^{2} \int_{\mathbb{R}^{3}} \int_{\mathbb{S}_{+}^{2}} M_{j}^{1 / 2}(w) M_{k}^{1 / 2}\left(v^{\odot}\right) \widehat{h}_{l}\left(w^{\odot}\right)\langle\varepsilon, v-w\rangle d \varepsilon d w .
\end{aligned}
$$

We obtain

$$
\begin{aligned}
& \mathscr{R}_{i}^{(2)}(\underline{\widehat{h}})=\int_{\mathbb{R}^{3}} \Upsilon_{i j}^{(2)}(v, w) \widehat{h}_{k}(w) d w, \\
& \mathscr{R}_{i}^{(3)}(\underline{\widehat{h}})=\int_{\mathbb{R}^{3}} \Upsilon_{i j}^{(3)}(v, w) \widehat{h}_{l}(w) d w .
\end{aligned}
$$

with the kernels given as follows. For the operator $\mathscr{R}_{i}^{(2)}$ the calculations are simple and

$$
\begin{aligned}
\Upsilon_{i j}^{(2)}(v, w)= & \alpha_{R} \sigma_{i j}^{2} \sqrt{n_{j} n_{l}}\left(\frac{m_{j}}{2 \pi k T}\right)^{1 / 2}\left(\frac{m_{i}}{2 \mu_{i j}}\right)^{2} \frac{1}{\|v-w\|} \\
& \times \exp \left[-\frac{m_{j}}{8 k T} \frac{\left(v^{2}-w^{2}\right)^{2}}{\|v-w\|^{2}}-\frac{m_{j}}{8 k T}\left(\frac{m_{i}}{m_{j}}\right)^{2}(v-w)^{2}\right] .
\end{aligned}
$$

Concerning the kernel of the operator $\mathscr{R}_{i}^{(3)}$, similarly to what we have done before for the elastic operator $\mathscr{Q}_{i}^{(3)}$, we consider three different cases. If $m_{j}=m_{i}$, and therefore all constituents have equal molecular masses, then the kernel is given by

$$
\begin{aligned}
\Upsilon_{i j}^{\left(3^{0}\right)}(v, w)= & \alpha_{R} \sigma_{i j}^{2}\left(n_{j} n_{k}\right)^{1 / 2}\left(\frac{m_{i}}{2 \pi k T}\right)^{1 / 2} \frac{1}{\|v-w\|} \\
& \times \exp \left[-\frac{m_{i}}{8 k T} \frac{\left(v^{2}-w^{2}\right)^{2}}{\|v-w\|^{2}}-\frac{m_{i}}{8 k T}(v-w)^{2}\right] .
\end{aligned}
$$

If $m_{j}>m_{i}$, the kernel is given by 


$$
\begin{aligned}
\Upsilon_{i j}^{\left(3^{+}\right)}(v, w)= & \frac{2 \pi \alpha_{R} \sigma_{i j}^{2}}{\left(\frac{m_{j}-m_{i}}{m_{i}+m_{j}}\right)^{2}\left(n_{j} n_{k}\right)^{1 / 2}\left(\frac{m_{i} m_{j}}{(2 \pi k T)^{2}}\right)^{3 / 4}\|v-w\|} \\
& \times \exp \left[-\frac{1}{4 k T} \frac{\left(m_{j} w-m_{i} v\right)^{2}}{m_{i}+m_{j}}\right] \\
& \times \int_{0}^{\pi / 2} \cos ^{2} \theta \exp \left[-\frac{\mu_{i j}}{k T}\left(\frac{\|v+w\|}{2}-\frac{\|v-w\| \cos \theta}{\frac{m_{j}-m_{i}}{m_{i}+m_{j}}}\right)^{2}\right. \\
& \left.\left.+\frac{\|v-w\|\|v+w\| \cos \theta\left(1-\cos \theta^{\star}\right)}{\frac{m_{j}-m_{i}}{m_{i}+m_{j}}}\right)\right] d \theta .
\end{aligned}
$$

If $m_{j}<m_{i}$, the kernel $\Upsilon_{i j}^{\left(3^{-}\right)}$is given by a similar expression to (52), but with the integral in $\theta$ extended to $[\pi / 2, \pi]$ instead of $[0, \pi / 2]$. In expressions (50), (51) and (52), the indices $i, j, k, l$ are from the set $\{(1,2,3,4),(2,1,4,3),(3,4,1,2),(4,3,2,1)\}$.

\subsection{Properties of the kernels}

From the explicit representation of the kernels given in the previous section, it is easy to state the properties given below.

Proposition 5 For any $i, s=1,2,3,4$, the following properties of the elastic kernels $K_{i s}^{(\alpha)}, \alpha=1,2,3$, hold for all $v, w$ a.e. in $\mathbb{R}^{3}$ :
(a) $K_{i s}^{(1)}(v, w)=K_{s i}^{(1)}(w, v)$;
(c) $K_{i s}^{\left(3^{0}\right)}(v, w)=K_{s i}^{\left(3^{0}\right)}(w, v)$;
(b) $K_{i s}^{(2)}(v, w)=K_{i s}^{(2)}(w, v)$;
(d) $K_{i s}^{\left(3^{+}\right)}(v, w)=K_{s i}^{\left(3^{-}\right)}(w, v)$.

The properties stated in Proposition 5 assure the symmetry of the elastic integral operators $\mathscr{Q}_{i}^{(\alpha)}(\alpha=1,2,3)$. See expressions (32), (33), (37), (38) and (39).

Similar properties hold for the kernels $N_{i s}^{(\alpha)}$ of the elastic operators $\mathscr{T}_{i}^{(\alpha)}(\alpha=2,3)$, assuring the symmetry of these operators.

Proposition 6 For any $(i, j) \in\{(1,2),(2,1),(3,4),(4,3)\}$, the following properties of the reactive kernels $\Upsilon_{i j}^{(\alpha)}, \alpha=2,3$, hold for all $v, w$ a.e. in $\mathbb{R}^{3}$ :

(a) $\Upsilon_{i j}^{(2)}(v, w)=r_{i j}^{(2)}(w, v) ; \quad$ (c) $\Upsilon_{i j}^{\left(3^{+}\right)}(v, w)=r_{j i}^{\left(3^{-}\right)}(w, v)$.

(b) $\Upsilon_{i j}^{\left(3^{0}\right)}(v, w)=\Upsilon_{j i}^{\left(3^{0}\right)}(w, v)$;

The properties stated in Proposition 6 assure the symmetry of the reactive integral operators $\mathscr{R}_{i}^{(\alpha)}(\alpha=2,3)$. See expressions (48) and (49). 


\section{Compactness of the linearized collisional operator}

In this section, we study the compactness of the elastic and reactive linearized operators, as operators from $\left(L^{2}\left(\mathbb{R}^{3}\right)\right)^{4}$ to $\left(L^{2}\left(\mathbb{R}^{3}\right)\right)^{4}$. We introduce the notation

$$
\begin{array}{ll}
\mathscr{Q}^{(\alpha)}=\left(\mathscr{Q}_{1}^{(\alpha)}, \mathscr{Q}_{2}^{(\alpha)}, \mathscr{Q}_{3}^{(\alpha)}, \mathscr{Q}_{4}^{(\alpha)}\right), \quad \alpha=1,2,3, \\
\mathscr{T}^{(\alpha)}=\left(\mathscr{T}_{1}^{(\alpha)}, \mathscr{T}_{2}^{(\alpha)}, \mathscr{T}_{3}^{(\alpha)}, \mathscr{T}_{4}^{(\alpha)}\right), & \alpha=2,3 \\
\mathscr{R}^{(\alpha)}=\left(\mathscr{R}_{1}^{(\alpha)}, \mathscr{R}_{2}^{(\alpha)}, \mathscr{R}_{3}^{(\alpha)}, \mathscr{R}_{4}^{(\alpha)}\right), & \alpha=2,3 .
\end{array}
$$

We extend to our mixture of reactive gases the techniques introduced by Grad (see $[12,13])$ and then revisited by Cercignani, Illner and Pulvirenti (see [14]), for one single inert gas. However, these techniques seem to be not enough for the operators $\mathscr{Q}^{(3)}, \mathscr{T}^{(3)}$ and $\mathscr{R}^{(3)}$, due to the cumbersome expressions of their terms when the molecular masses are different. See expressions (41), (42) and (52). For these operators, our idea is to adapt to our model the methodology recently proposed by Boudin, Grec, Pavić and Salvarani (see [15]) for a mixture of non-reactive gases. In paper [15], the authors consider the non-reactive Boltzmann equation for gas mixtures and propose a new approach to treat the terms of the linearized operators associated with particles with different masses.

Work is in progress to extend the approach of paper [15] to the similar terms appearing in our model. In the present paper we focus on the compactness of both the elastic operator $\mathscr{Q}^{(1)}$ and the reactive operator $\mathscr{R}^{(2)}$. Operators $\mathscr{Q}^{(2)}$ and $\mathscr{T}^{(2)}$ can be treated in a similar way to $\mathscr{R}^{(2)}$.

\subsection{Compactness of the operator $\mathscr{Q}^{(1)}$}

We consider the elastic kernel $K_{i s}^{(1)}$ of the operator $\mathscr{Q}^{(1)}$ defined by expression (34). We start with the estimate for for $K_{i s}^{(1)}$ stated in the following lemma.

Lemma 3. For each $i, s=1,2,3,4$, the elastic kernel $K_{i s}^{(1)}$ is square integrable with respect to $w$. We have

$$
\int_{\mathbb{R}^{3}}\left(K_{i s}^{(1)}\right)^{2}(v, w) d w \leq C\left(1+\|v\|^{2}\right) \exp \left(-\frac{m_{i} v^{2}}{2 k T}\right)
$$

where $C$ depends only on $m_{i}, n_{i}, \sigma_{i s}$, and $T$.

Proof. For $i, s=1,2,3,4$, we have 


$$
\begin{aligned}
& \int_{\mathbb{R}^{3}}\left(K_{i s}^{(1)}\right)^{2}(v, w) d w= \pi^{2} \sigma_{i s}^{4} n_{i} n_{s}\left(\frac{m_{i} m_{s}}{(2 \pi k T)^{2}}\right)^{\frac{3}{2}} \exp \left(-\frac{m_{i} v^{2}}{2 k T}\right) \\
& \quad \times \int_{\mathbb{R}^{3}}|v-w|^{2} \exp \left(-\frac{m_{s} w^{2}}{2 k \mathscr{T}}\right) d w \\
& \leq 2 \pi^{2} \sigma_{i s}^{4} n_{i} n_{s}\left(\frac{m_{i} m_{s}}{(2 \pi k T)^{2}}\right)^{\frac{3}{2}} \exp \left(-\frac{m_{i} v^{2}}{2 k T}\right) \\
& \quad \times \int_{\mathbb{R}^{3}}\left(|v|^{2}+|w|^{2}\right) \exp \left(-\frac{m_{s} w^{2}}{4 k T}\right) d w \\
& \leq C\left(1+|v|^{2}\right) \exp \left(-\frac{m_{i} v^{2}}{2 k T}\right)
\end{aligned}
$$

and the proof is complete.

Then we can prove the compactness of $\mathscr{Q}^{(1)}$, as follows.

Theorem 1 The linearized elastic operator $\mathscr{Q}^{(1)}$ is a compact operator from $\left(L^{2}\left(\mathbb{R}^{3}\right)\right)^{4}$ to $\left(L^{2}\left(\mathbb{R}^{3}\right)\right)^{4}$.

Proof. It is enough to show that each $\mathscr{Q}_{i}^{(1)}(i=1,2,3,4)$ is a compact operator from $\left(L^{2}(\mathbb{R})\right)^{4}$ to $L^{2}(\mathbb{R})$. To this end, we notice that, for fixed $i$ and $s$, the operator $A$ with the kernel $K_{i s}^{(1)}$ given by (34) is a compact operator from $L^{2}\left(\mathbb{R}^{3}\right)$ to $L^{2}\left(\mathbb{R}^{3}\right)$. Indeed, condition (54) implies that $K_{i s}^{(1)} \in L^{2}\left(\mathbb{R}^{3} \times \mathbb{R}^{3}\right)$, showing that the operator $A$, as a Hilbert-Schmidt operator, is compact from $L^{2}\left(\mathbb{R}^{3}\right)$ to $L^{2}\left(\mathbb{R}^{3}\right)$. Since this is true for each $s=1,2,3,4$, the operator $\mathscr{Q}_{i}^{(1)}(i=1,2,3,4)$ is a compact operator from $\left(L^{2}\left(\mathbb{R}^{3}\right)\right)^{4}$ to $L^{2}\left(\mathbb{R}^{3}\right)$, and this completes the proof.

\subsection{Compactness of the operator $\mathscr{R}^{(2)}$}

Similarly to what we have done in the previous subsection, we consider the reactive kernel $\Upsilon_{i j}^{(2)}$ of the operator $\mathscr{R}^{(2)}$ defined by expression (50). We first state the following result.

Proposition 7 For each $i, j=1,2,3,4$, the reactive kernel $\Upsilon_{i j}^{(2)}$ is square integrable with respect to $w$. Moreover, we have

$$
\left\|r_{i j}^{(2)}(v, \cdot)\right\|_{L^{2}\left(\mathbb{R}^{3}\right)} \leq C,
$$

where $C$ is independent of $v$. 
Proof. From

$$
\begin{aligned}
\Upsilon_{i j}^{(2)}(v, w)=\alpha_{R} \sigma_{i j}^{2} & \sqrt{n_{j} n_{l}}\left(\frac{m_{j}}{2 \pi k T}\right)^{1 / 2}\left(\frac{m_{i}}{2 \mu_{i j}}\right)^{2} \frac{1}{\|v-w\|} \\
& \times \exp \left[-\frac{m_{j}}{8 k T} \frac{\left(v^{2}-w^{2}\right)^{2}}{\|v-w\|^{2}}-\frac{m_{j}}{8 k T}\left(\frac{m_{i}}{m_{j}}\right)^{2}(v-w)^{2}\right] \\
\leq \alpha_{R} \sigma_{i j}^{2} & \sqrt{n_{j} n_{l}}\left(\frac{m_{j}}{2 \pi k T}\right)^{1 / 2}\left(\frac{m_{i}}{2 \mu_{i j}}\right)^{2} \frac{1}{\|v-w\|} \\
& \times \exp \left[-\frac{m_{j}}{8 k T}\left(\frac{m_{i}}{m_{j}}\right)^{2}(v-w)^{2}\right],
\end{aligned}
$$

and for any $M>0$, we obtain

$$
\begin{aligned}
\int_{\mathbb{R}^{3}}\left(Y_{i j}^{(2)}\right)^{2}(v, w) d w \leq & C_{1} \int_{|w| \leq M} \frac{1}{\|v-w\|^{2}} d w \\
& +C_{2} \int_{|w|>M} \exp \left[-\frac{m_{j}}{8 k T}\left(\frac{m_{i}}{m_{j}}\right)^{2}(v-w)^{2}\right] d w<C .
\end{aligned}
$$

The first term on the right hand side of (57) is bounded since $1 /\|v-w\|^{2}$ is integrable on any bounded set containing $v$, while the second term is bounded because $\exp \left(-c x^{2}\right)$ is integrable over $\mathbb{R}^{3}$ for any $c>0$. The constant $C$ does not depend on $v$ since the expression in (57) is translational invariant.

We also have the following estimate for $\Upsilon_{i j}^{(2)}$.

Proposition 8 For each $i, j=1,2,3,4$, the reactive kernel $\Upsilon_{i j}^{(2)}$ is integrable with respect to $w$, and we have

$$
\int_{\mathbb{R}^{3}} \Upsilon_{i j}^{(2)}(v, w) d w \leq \frac{C}{\left(1+\|v\|^{2}\right)^{1 / 2}},
$$

where $C$ is independent of $v$.

Proof. See [13] or [14].

Then we can prove the compactness of $\mathscr{R}^{(2)}$, as follows.

Theorem 2 The linearized reactive operator $\mathscr{R}^{(2)}$ is a compact operator from $\left(L^{2}\left(\mathbb{R}^{3}\right)\right)^{4}$ to $\left(L^{2}\left(\mathbb{R}^{3}\right)\right)^{4}$.

Proof. It is enough to show that each $\mathscr{R}_{i}^{(2)}(i=1,2,3,4)$ is a compact operator from $\left(L^{2}\left(\mathbb{R}^{3}\right)\right)^{4}$ to $L^{2}\left(\mathbb{R}^{3}\right)$. For fixed $i$ and $j$, consider the operator $A$ with the kernel $\Upsilon_{i j}^{(2)}$ given by (50). 
For $M>0$, define $\chi_{M}$ to be the characteristic function of the set $\left\{v \in \mathbb{R}^{3}:|v| \leq M\right\}$. Consider the identity $A f=\chi_{M} A f+\left(1-\chi_{M}\right) A f$ for $f \in L^{2}\left(\mathbb{R}^{3}\right)$. Proposition $7 \mathrm{im}$ plies that for each $M>0$, the operator $\chi_{M} A$, as a Hilbert-Schmidt operator, is compact from $L^{2}\left(\mathbb{R}^{3}\right)$ to $L^{2}\left(\mathbb{R}^{3}\right)$. Now, if $\|\cdot\|_{B\left(L^{2}\left(\mathbb{R}^{3}\right)\right)}$ denotes the operator norm in the space of bounded operators on $L^{2}\left(\mathbb{R}^{3}\right)$, then using Schwartz inequality, Propositions 7 and 8 , the operator $\left(1-\chi_{M}\right) A$ has the property (see [14], Theorem 7.2.4),

$$
\begin{aligned}
& \left\|\left(1-\chi_{M}\right) A\right\|_{B\left(L^{2}\left(\mathbb{R}^{3}\right)\right)}^{2}=\sup _{\|f\|_{L^{2}\left(\mathbb{R}^{3}\right)} \leq 1} \int_{\mathbb{R}^{3}}\left(1-\chi_{M}(v)\right)\left[\int_{\mathbb{R}^{3}} \Upsilon_{i j}^{(2)}(v, w) f(w) d w\right]^{2} d v \\
& \leq \sup _{\|f\|_{L^{2}\left(\mathbb{R}^{3}\right)} \leq 1} \int_{\mathbb{R}^{3}}\left(1-\chi_{M}(v)\right)\left[\int_{\mathbb{R}^{3}} \Upsilon_{i j}^{(2)}(v, w) d w\right] \\
& \times\left[\int_{\mathbb{R}^{3}} r_{i j}^{(2)}(v, w)(f(w))^{2} d w\right] d v \\
& \leq C \sup _{\|f\|_{L^{2}\left(\mathbb{R}^{3}\right)} \leq 1} \int_{\mathbb{R}^{3}}\left(1-\chi_{M}(v)\right)\left(1+\|v\|^{2}\right)^{-1 / 2} \\
& \times\left[\int_{\mathbb{R}^{3}} Y_{i j}^{(2)}(v, w)(f(w))^{2} d w\right] d v \\
& \leq C\left(1+M^{2}\right)^{-1 / 2} \sup _{\|f\|_{L^{2}(\mathbb{R})} \leq 1} \int_{\|v\|>M}\left[\int_{\mathbb{R}^{3}} r_{i j}^{(2)}(v, w)(f(w))^{2} d w\right] d v \\
& \leq C\left(1+M^{2}\right)^{-1 / 2} \sup _{\|f\|_{L^{2}\left(\mathbb{R}^{3}\right)} \leq 1} \int_{\mathbb{R}^{3}}\left[\int_{\mathbb{R}^{3}} \Upsilon_{i j}^{(2)}(v, w)(f(w))^{2} d w\right] d v \\
& \leq C\left(1+M^{2}\right)^{-1 / 2} \sup _{\|f\|_{L^{2}\left(\mathbb{R}^{3}\right)} \leq 1} \int_{\mathbb{R}^{3}}(f(w))^{2} d w \leq C\left(1+M^{2}\right)^{-1 / 2} .
\end{aligned}
$$

Therefore, $\left(1-\chi_{M}\right) A \rightarrow 0$ in the operator norm as $M \rightarrow \infty$. This also yields that $\chi_{M} A \rightarrow A$ in the operator norm as $M \rightarrow \infty$. Since for each $M>0, \chi_{M} A$ is a compact operator from $L^{2}\left(\mathbb{R}^{3}\right)$ to $L^{2}\left(\mathbb{R}^{3}\right)$, and the set of compact operators is closed in the space of bounded operators with the operator norm $\|\cdot\|_{B\left(L^{2}\left(\mathbb{R}^{3}\right)\right)}$, we conclude that $A$ is compact as an operator from $L^{2}\left(\mathbb{R}^{3}\right)$ to $L^{2}\left(\mathbb{R}^{3}\right)$. Since this is true for each $j=1,2,3,4$, the operator $\mathscr{R}_{i}^{(2)}(i=1,2,3,4)$ is a compact operator from $\left(L^{2}\left(\mathbb{R}^{3}\right)\right)^{4}$ to $L^{2}\left(\mathbb{R}^{3}\right)$. Thus, the operator $\mathscr{R}^{(1)}$ is compact from $\left(L^{2}\left(\mathbb{R}^{3}\right)\right)^{4}$ to $\left(L^{2}\left(\mathbb{R}^{3}\right)\right)^{4}$ and the proof is complete.

Acknowledgements The paper is partially supported by the Research Centre of Mathematics of the University of Minho, through the National Funds from the "Fundação para a Ciência e a Tecnologia”, Project PEstOE/MAT/UI0013/2014. 


\section{References}

1. Polewczak, J., Soares, A.J.: Kinetic Theory of Simple Reacting Spheres I. In: 27th International Symposium on Rarefied Gas Dynamics, 2010, Pacific Grove. AIP Conference Proceedings, vol. 1333, pp. 117-122 (2011)

2. Carvalho, F., Polewczak, J., Soares, A.J.: On the kinetic systems for simple reacting spheres: modeling and linearized equations. In: Particle Systems and Partial Differential Equations, 2012, Braga. Springer Proceedings in Mathematics \& Statistics, vol. 75, pp. 251-267 (2014)

3. Marron, M. T.: Simple Collision Theory of Reactive Hard Spheres. J. Chem. Phys. 52, 4060$4061(1970)$

4. Xystris, N., Dahler, J. S.: Mass and momentum transport in dilute reacting gases. J. Chem. Phys. 68, 354-373 (1978)

5. Xystris, N., Dahler, J.S.: Kinetic theory of simple reacting spheres. J. Chem. Phys. 68, 387$401(1978)$

6. Dahler, J.S., Qin, L.: The kinetic theory of a simple reacting fluid: Scattering functions and relaxation processes. J. Chem. Phys. 103, 725-750 (1995)

7. Polewczak, J.: The Kinetic theory of simple reacting Spheres: I. Global Existence Result in a Dilute-Gas Case. J. Stat. Phys. 100, 327-362 (2000)

8. Prigogine, I., Xhrouet, E.: On the perturbation of Maxwell distribution function by chemical reaction in gases. Physica XV, 913-932 (1949)

9. Prigogine, I., Mahieu, M.: Sur La Perturbation De La Distribution De Maxwell Par Des Réactions Chimiques En Phase Gazeuse. Physica XVI, 51-64 (1950)

10. Shizgal, B., Karplus, M.: Nonequilibrium contributions to the rate of reaction. I. Perturbation of the Velocity Distribution Function. J. Chem. Phys. 52, 4262-4278 (1970)

11. Carvalho, F.: Mathematical methods for the Boltzmann equation in the context of chemically reactive gases, Doctoral Thesis, 2012, http://hdl. handle.net/1822/24430 Repositorium of the University of Minho.

12. Grad, H.: Asymptotic theory of the Boltzmann Equation, Phys. Fluids, 6, 147-181 (1963)

13. Grad, H.: Asymptotic theory of the Boltzmann Equation II, Rarefied Gas Dynamics, Volume 1, Proc. 3rd Int. Symp., Ed. J. A. Laurmann, New York: Academic Press, $26-59$ (1963)

14. C. Cercignani, R. Illner, and M. Pulvirenti, The Mathematical Theory of Dilute Gases, Springer-Verlag, New York, (1994).

15. Boudin, L., Grec, B., Pavić, M., and Salvarani, F.: Diffusion Asymptotics of a kinetic model for gaseous mixtures, Kinetic and Related Models, 6, 137-157 (2013) 